\title{
Comunicación
}

\section{Cinética y morfometría espermática en semen congelado sexado y convencional de toros Brown Swiss}

\author{
Kinetics and sperm morphometry in sexed and conventional frozen semen \\ of Brown Swiss bulls
}

\author{
Edwin Ormachea V., ${ }^{1,4}$, Bilo Calsin C. ${ }^{2}$, Enrique Zegarra O. $^{3}$
}

\section{Resumen}

\begin{abstract}
El estudio tuvo como objetivo determinar la cinética y morfometría espermática de semen congelado sexado y convencional de toros Brown Swiss utilizando el sistema Integrated Sperm Analisys. Se utilizaron 10 pajillas de semen sexado y 10 pajillas de semen convencional de origen americano. Los valores de la cinética (velocidad curvilínea [VCL], velocidad media [VAP], velocidad rectilínea [VSL], amplitud del desplazamiento lateral de cabeza [ALH], frecuencia de batido [BCF], índice de linealidad [LIN[, índice de rectitud [STR], índice de oscilación [WOB]) y morfometría espermática (área, largo, ancho y perímetro de la cabeza de los espermatozoides) fueron similares entre el semen sexado y convencional de toros Brown Swiss.
\end{abstract}

Palabras clave: cinética; morfometría; semen sexado; semen congelado

\section{Abstract}

The aim of this study was to determine the kinetics and sperm morphometry in sexed and conventional frozen semen of Brown Swiss bulls using the Integrated Sperm Analisys system. Ten straws of sexed semen and 10 straws of conventional semen imported from USA were used. The values of kinetics (curvilinear velocity [VCL], average velocity [VAP], rectilinear velocity [VSL], amplitude of lateral head displacement [ALH], beat frequency $[\mathrm{BCF}]$, linearity index [LIN [, index of straightness [STR], oscillation index

\footnotetext{
${ }^{1}$ Departamento Académico de Medicina Veterinaria y Zootecnia, Universidad Nacional Amazónica Madre de Dios, Perú

${ }^{2}$ Facultad de Medicina Veterinaria y Zootecnia, Universidad Nacional del Altiplano - UNA, Puno, Perú

${ }^{3}$ Expermex EIRL, Perú

${ }^{4}$ E-mail: edwinormachea@gmail.com
}

Recibido: 31 de mayo de 2018

Aceptado para publicación: 27 de octubre de 2018 
[WOB]) and sperm morphometry (area, length, width and perimeter of the head of the sperm) were similar between sexed and conventional semen of Brown Swiss bulls frozen in straws.

Key words: kinetics; morphometry; sexed semen; frozen semen

\section{INTRODUCCIÓN}

La inseminación artificial es una técnica ampliamente utilizada en Puno, Perú, para la mejora de la respuesta productiva del ganado bovino. La evaluación de la calidad del semen permite obtener una expresión más precisa de la fisiología o de las alteraciones del aparato reproductor masculino, particularmente en el proceso de la espermatogénesis (Kruger et al., 1988; Tamayo Torres, 2013).

La evaluación de la morfología espermática de manera visual conlleva a la obtención de valores susceptibles de imprecisión, dependiendo del técnico y de otros factores, de allí que los resultados que aporta el espermiograma no sea hasta el momento, por esta y otras razones, un buen predictor de la fertilidad (Quintero-Moreno et al., 2007). Se han desarrollado nuevas tecnologías in vitro, como la citometría de flujo o los sistemas CASA (Computer Assisted Sperm Analisis), que permiten el estudio de múltiples características funcionales y morfológicas de los espermatozoides para intentar predecir la capacidad fecundante del semen de la forma más objetiva posible (WHO, 1999). Además, la aplicación de estas nuevas técnicas permite la reevaluación de los protocolos de criopreservación, tales como la elección del mejor diluyente para obtener una mayor supervivencia espermática posdescongelación o la temperatura ideal de descongelación de las dosis seminales (Muiño et al., 2005).

El uso de los sistemas CASA e ISAS (Integrated Sperm Analysis System) proporciona una serie de datos relativos a la veloci- dad y trayectoria de cada espermatozoide individual, que permite identificar la existencia de subpoblaciones espermáticas con distintos patrones de movimiento que coexisten en la misma muestra de semen (Davis y Siemers, 1995; Holt et al., 1996; Abaigar et al., 1999; ; Rigau et al., 2001; Quintero-Moreno et al., 2004), lo cual proporciona una visión más realista que la motilidad media de la muestra.

\section{MATERIALES Y MÉTODOS}

El análisis de la cinética y morfometría espermática de semen congelado se realizó en el laboratorio de reproducción de la Universidad Nacional San Antonio Abad del Cusco, empleando el Integrated Semen Analysis System - IISAS ${ }^{\circledR}$ v. 1.2, el cual emplea un microscopio UOP-UB200i (Proiser R+D, Paterna, Valencia, España).

Para la cinética espermática se determinaron la velocidad curvilínea, $\operatorname{VCL}(\mu \mathrm{m} / \mathrm{s})$, velocidad media, VAP $(\mu \mathrm{m} / \mathrm{s})$, velocidad rectilínea, VSL $(\mu \mathrm{m} / \mathrm{s})$, amplitud del desplazamiento lateral de cabeza, $\operatorname{ALH}(\mu \mathrm{m} / \mathrm{s})$, frecuencia de batido, BCF $(\mathrm{Hz})$, índice de linealidad, LIN (\%), índice de rectitud, STR (\%) e índice de oscilación, WOB (\%), de los espermatozoides en semen congelado de 10 pajillas sexadas de $0.25 \mathrm{ml}$ y 10 pajillas convencionales de $0.5 \mathrm{ml}$ de toros Brown Swiss de origen americano (adquiridos de la empresa Expermex EIRL, Perú).

Las pajillas de semen fueron descongeladas a $37^{\circ} \mathrm{C}$ en baño maría durante 30 segundos. Se tomó una muestra de $5 \mu 1$ de cada pajilla y se colocó en una placa Petri. Fue 
observada con un lente de 10X de contraste en fase negativo y platina térmica $\left(37^{\circ} \mathrm{C}\right)$. La señal del video se registró con una video cámara Proiser 782C donde se capturaron 25 imágenes por segundo, analizando los espermatozoides en la configuración establecida de acuerdo con la clasificación por Muiño et al. (2006).

Se prepararon frotis con $5 \mu 1$ de semen descongelado y fueron teñidos con DiffQuick (Siemens Healthcare Diagnostics Products). Las muestras teñidas se evaluaron utilizando el módulo de morfometría del ISAS, empleando el lente de inmersión 100X de un microscopio de contraste de fases y fluorescencia UB200i con cámara. El sistema delineó los bordes de los espermatozoides fotografiados. La búsqueda y captura de las imágenes de espermatozoides se realizó de manera manual. Se determinó el área $\left(\mu \mathrm{m}^{2}\right)$, largo $(\mu \mathrm{m})$, ancho $(\mu \mathrm{m})$ y perímetro $(\mu \mathrm{m})$ de la cabeza de los espermatozoides.

Para el análisis estadístico se utilizó la prueba de Kolmogorov-Smirnov para determinar la distribución normal y homogénea de los datos. Los datos expresados en porcentajes de los parámetros cinéticos de LIN=Índice de linealidad, STR=Índice de rectitud y WOB=Índice de oscilación fueron sujetos a una transformación de raíz cuadrada. El análisis se hizo mediante un análisis de varianza de medidas repetidas. Todos los análisis estadísticos se realizaron utilizando el programa SPSS 22 (SPSS Inc, EEUU).

\section{Resultados}

\section{Cinética Espermática}

No se encontraron diferencias significativas en los parámetros de cinética espermática entre el semen sexado y convencional (Cuadro 1), aunque se puede observar que los espermatozoides de las pajillas descongeladas de semen sexado presenta- ban movimientos ligeramente más rápidos, de mayor velocidad y de trayectoria más rectilínea (VCL, VAP, VSL, BCF y ALH) que aquellos en pajillas con semen convencional.

\section{Morfometría Espermática}

No hubo diferencias significativas en los parámetros de morfometría espermática entre el semen sexado y semen convencional (Cuadro 2); sin embargo, se evidencian ligeros incrementos en la morfometría de la cabeza del espermatozoide de las pajillas de semen sexado con relación al de las pajillas con semen convencional.

\section{Discusión}

\section{Cinética Espermática}

Las medias encontradas en los parámetros de cinética espermática en semen sexado y convencional son ligeramente superiores a los valores encontrados por Ancco et al. (2014), quienes trabajando con ocho pajillas congeladas de toros con fertilidad conocida encontró los siguientes valores cinéticos espermáticos posdescongelación $(\mathrm{VCL}=73.16 \mu \mathrm{m} / \mathrm{s} ; \mathrm{VSL}=26.09 \mu \mathrm{m} / \mathrm{s}$; $\mathrm{VAP}=41.38 \mu / \mathrm{s} ; \mathrm{LIN}=35.76 \% ; \mathrm{STR}=$ $63.05 \%$; $\mathrm{BCF}=8.06 \mathrm{~Hz}$ ). Al respecto, Muiño (2007) indica que la raza, edad y la interacción raza $x$ edad tuvieron efectos significativos $(p<0.001)$ sobre los parámetros cinéticos, donde los espermatozoides de toros frisones presentaron movimientos de mayor velocidad y de trayectoria más rectilínea en comparación aquellos de eyaculados de toros Rubia Gallega. Así mismo, Hoflack et al. (2007) demostraron diferencias significativas en velocidad y linealidad de las trayectorias espermáticas a favor de semen de toros Holstein en comparación con semen de toros Azul Belga; hallazgos que sugieren que un componente genético podría ser la base de dichas diferencias. 
Cuadro 1. Parámetros de cinética espermática de pajillas de semen bovino Brown Swiss sexado $(n=10)$ y convencional $(n=10)$ utilizando el sistema IISAS ${ }^{\circledR}$

\begin{tabular}{lcccc}
\hline \multirow{2}{*}{ Cinética espermática } & \multicolumn{2}{c}{ Semen sexado } & \multicolumn{2}{c}{ Semen convencional } \\
\cline { 2 - 5 } & Promedio & D.E. & Promedio & D.E. \\
\hline VCL $(\mu \mathrm{m} / \mathrm{s})$ & $85.46^{\mathrm{a}}$ & 15.6 & $77.30^{\mathrm{a}}$ & 13.5 \\
VAP $(\mu \mathrm{m} / \mathrm{s})$ & $49.84^{\mathrm{a}}$ & 5.1 & $42.30^{\mathrm{a}}$ & 6.4 \\
VSL $(\mu \mathrm{m} / \mathrm{s})$ & $38.34^{\mathrm{a}}$ & 2.9 & $29.00^{\mathrm{a}}$ & 4.9 \\
BCF $(\mathrm{Hz})$ & $19.96^{\mathrm{a}}$ & 9.5 & $10.12^{\mathrm{a}}$ & 1.3 \\
ALH $(\mu \mathrm{m} / \mathrm{s})$ & $4.12^{\mathrm{a}}$ & 1.2 & $3.24^{\mathrm{a}}$ & 0.5 \\
LIN $(\%)$ & $46.32^{\mathrm{a}}$ & 10.6 & $39.07^{\mathrm{a}}$ & 11.4 \\
STR $(\%)$ & $77.48^{\mathrm{a}}$ & 8.7 & $68.70^{\mathrm{a}}$ & 8.5 \\
WOB $(\%)$ & $59.02^{\mathrm{a}}$ & 6.0 & $55.26^{\mathrm{a}}$ & 7.2 \\
\hline
\end{tabular}

VCL=Velocidad curvilínea; $\mathrm{VAP}=$ Velocidad media; $\mathrm{VSL}=$ Velocidad rectilínea; $\mathrm{BCF}=$ frecuencia de batido; $\mathrm{ALH}=A m p l i t u d$ media del desplazamiento lateral de la cabeza; LIN=Índice de linealidad; STR= Índice de rectitud; WOB =Índice de oscilación

Superíndices iguales dentro de filas indican que los valores fueron estadísticamente similares

Cuadro 2. Parámetros de morfometría de la cabeza del espermatozoide $(\mu \mathrm{m})$ de toros Brown Swiss en pajillas de semen sexado $(n=10)$ y convencional $(n=10)$ utilizando el sistema IISAS ${ }^{\circledR}$

\begin{tabular}{lcccc}
\hline \multirow{2}{*}{ Morfometría espermática } & \multicolumn{2}{c}{ Semen sexado } & \multicolumn{2}{c}{ Semen convencional } \\
\cline { 2 - 5 } & Promedio & D.E. & Promedio & D.E. \\
\hline Área $\left(\mu \mathrm{m}^{2}\right)$ & $21.51^{\mathrm{a}}$ & 1.87 & $18.42^{\mathrm{a}}$ & 2.33 \\
Largo $(\mu \mathrm{m})$ & $6.36^{\mathrm{a}}$ & 0.55 & $5.23^{\mathrm{a}}$ & 0.71 \\
Ancho $(\mu \mathrm{m})$ & $3.42^{\mathrm{a}}$ & 0.38 & $2.67^{\mathrm{a}}$ & 0.49 \\
Perímetro $(\mu \mathrm{m})$ & $17.94^{\mathrm{a}}$ & 0.99 & $14.51^{\mathrm{a}}$ & 2.58 \\
\hline
\end{tabular}

Superíndices iguales dentro de filas indican que los valores fueron estadísticamente similares

Se puede indicar que la velocidad media (VAP) y curvilínea (VCL) son reconocidas como parámetros importantes en la evaluación del semen, debido a que son los responsables del transporte de espermatozoides a lo largo del tracto genital de la hembra (Verstegen et al., 2002; Robayo et al., 2008). Verstegen et al. (2002) correlacionaron los parámetros cinéticos de los espermatozoides con la tasa de fertilización, encontrando que los valores de VAP, VSL y VCL fueron significativamente mayores en muestras que producen más del $50 \%$ de ovocitos fertilizados que en aquellas donde la tasa de fertilización de ovocitos fue inferior al 50\%. Muestras con altos valores de VAP, VSL y VCL, así como de LIN y BCF presentan mejor migración y penetración en el moco cervical (Mortimer, 2000; Verstegen et al., 2002); en forma similar, Cox et al. (2006) reportan que espermatozoides caprinos con velocidad eficiente en la migración del moco cervical in vitro presentan valores de LIN $>50 \%$ y ALH $=4.8 \mathrm{ìm}$. 


\section{Morfometría Espermática}

Diversos estudios demuestran diferencias raciales con respecto a la morfología de la cabeza del espermatozoide (Dowsett y Knott, 1996) y la morfometría (Ball y Mohammed, 1995; Phetudomsinsuk et al., 2008) en el caballo y en otras especies animales (Aggarwal et al., 2007; Saravia et al., 2007); sin embargo, los autores de este estudio no han ubicado estudio alguno que compare tales diferencias entre las subpoblaciones morfométricas en bovinos.

El eyaculado del toro está compuesto por poblaciones heterogéneas de espermatozoides, de allí que se les puede agrupar en función de su morfometría (Rubio-Guillén et al., 2007). Nava-Trujillo y Quintero-Moreno (2017) agruparon tres subpoblaciones espermáticas con base a su morfometría: de cabeza pequeña (SP1) con un área de $23.69 \mu \mathrm{m}^{2}$, longitud $6.97 \mu \mathrm{m}$, ancho $3.86 \mu \mathrm{m}$ y perímetro $19.03 \mu \mathrm{m}$; los que tienen dimensiones intermedias (SP2) con un área de $28.51 \mu \mathrm{m}^{2}$, longitud $7.77 \mu \mathrm{m}$, ancho $4.18 \mu \mathrm{m}$ y perímetro $21.05 \mu \mathrm{m}$; y aquellos de mayor tamaño (SP3) con un área de $32.10 \mu \mathrm{m}^{2}$, longitud $8.30 \mu \mathrm{m}$, ancho $4.44 \mu \mathrm{m}$ y perímetro $22.57 \mu \mathrm{m}$, donde la subpoblación intermedia representaba el $60.5 \%$. Esta información concuerda, asimismo, con Rubio-Guillén et al. (2007), quienes, además, describen una población intermedia predominante de $50.4 \%$.

Con base a lo anterior, los espermatozoides de este estudio estarían mayormente agrupados como aquellos con cabeza pequeña (SP1); sin embargo, es posible que el proceso de criopreservación haya afectado su tamaño (Rubio-Guillén et al., 2007). Los espermatozoides difieren en la forma de la cabeza, lo cual influencia la valoración de la calidad seminal (Thurston et al., 2001). Así, se ha demostrado que la morfometría espermática influye en la calidad del movimiento espermático (Malo et al., 2006; Gil et al., 2009) y en la capacidad que presenta un eyaculado para responder a los distintos métodos de conservación (Esteso et al., 2006). Además, en otras especies como el caballo (Casey et al., 1997) y el cerdo (González Villalobos et al., 2008) se ha puesto en evidencia su implicancia en la fertilidad.

\section{Conclusiones}

Los valores de la cinética y morfometría espermática fueron similares entre el semen sexado y convencionales de toros Brown Swiss congelados en pajillas.

\section{Agradecimientos}

Los autores manifiestan su agradecimiento al MVZ Enrique Zegarra Ortiz por su apoyo en la realización del análisis de semen, y a la Municipalidad del distrito de Asillo por facilitar la ejecución del estudio en beneficio del sector agropecuario.

\section{Literatura Citada}

1. Abaigar T, Cano M, Pickard AR, Holt $W V .2001$. Use of computer-assisted sperm motility assessment and multivariate pattern analysis to characterize ejaculate quality in Mohor gazelles (Gazella dama mhorr): effects of body weight, electroejaculation technique and short-term semen storage. Reproduction 122: 265-273. doi: 10.1530/rep.0.1220265

2. Abaigar T, Holt WV, Harrison RAP, del Barrio G. 1999. Sperm subpopulations in boar (Sus scrofa) and gazelle (Gazella dama mhorr) semen as revealed by pattern analysis of computerassisted motility assessments. Biol Reprod 1: 32-41. doi: 10.1095/ biolreprod60.1.32

3. Aggarwal RAK, Ahlawat SPS, Kumar Y, Panwar PS, Singh K, Bhargava M. 2007. Biometry of frozen-thawed sperm from eight breeds of Indian buffaloes (Bubalus bubalis). Theriogenology 68: 682-686. doi: 10.1016/j.theriogenology.2007.03.028 
4. Ancco E, Quispe C, Mellisho E. 2014. Parámetros de cinética espermática antes y después de la selección con gradiente de Percoll 45/90 en semen congelado de bovinos. Spermova 4: 65-67.

5. Ball BA, Mohammed HO. 1995. Morphometry of stallion spermatozoa by computer-assisted image analysis. Theriogenology 44: 367-377. doi: 10.1016/0093-691X(95)00191-A

6. Casey PJ, Gravance CG, Davis RO, Chabot DD, Liu IKM. 1997. Morphometric differences in sperm head dimensions of fertile and subfertile stallions. Theriogenology 47: 575-582. doi: 10.1016/S0093-691X(97)00015-0

7. Cox JF, Alfaro V, Montenegro V, Rodríguez-Martínez H. 2006. Computer-assisted analysis of sperm motion in goats and its relationship with sperm migration in cervical mucus. Theriogenology 66: 860-867. doi: 10.1016/ j.theriogenology.2006.01.062

8. Davis RO, Siemers RJ. 1995. Derivation and reliability of kinematic measures of sperm motion. Reprod Fert Develop 7: 857-869. doi: 10.1071/ RD9950857

9. Dowsett KF, Knott LM. 1996. The influence of age and breed on stallion semen. Theriogenology 46: 397-412. doi: 10.1016/0093-691X(96)00162-8

10. Esteso MC, Fernández-Santos MR, Soler AJ, Montoro V, Quintero-Moreno A, Garde JJ. 2006. The effects of cryopreservation on the morphometric dimensions of Iberian red deer (Cervus elaphus hispanicus) epididymal sperm heads. Reprod Domest Anim 41: 241246. doi: $10.1111 / \mathrm{j} .1439-0531.2006$.00676.x

11. Gil MC, García-Herreros M, Barón FJ, Aparicio IM, Santos AJ, GarcíaMarín LJ. 2009. Morphometry of porcine spermatozoa and its functional significance in relation with the motility parameters in fresh semen. Theriogenology 71: 254-263. doi: 10.1016/ j.theriogenology.2008.07.007
12. González D, Quintero-Moreno A, Garde JJ, Esteso MC, FernándezSantos R, Rubio-Guillén M, et al. 2008. Morphometry characterization of boar sperm head with computer assisted analysis (preliminary results). Rev Cient 18: 570-577.

13. Hoflack G, Opsomer G, Rijsselaere T, Van Soom A, Maes D, de Kruif A, Duchateau L. 2007. Comparison of computer-assisted sperm motility analysis parameters in semen from Belgian Blue and Holstein-Friesian Bulls. Reprod Domest Anim 42: 153-161. doi: 10.1111/j.1439-0531.2006.00745.x

14. Holt C, Holt WV, Moore H. 1996. Choice of operating conditions to minimize sperm subpopulation sampling bias in the assessment of boar semen by computer-assisted semen analysis. J Androl 17: 587-596. doi: 10.1002/j.19394640.1996.tb01837.x

15. Kruger TF, Acosta AA, Simmons KF, Swanson RJ, Matta JF, Oehninger $S$. 1988. Predictive value of abnormal sperm morphology in in vitro fertilization. Fertil Steril 49: 112-117. doi: 10.1016/ S0015-0282(16)59660-5

16. Malo AF, Gomendio M, Garde J, Lang-Lenton B, Soler AJ, Roldan ER. 2006. Sperm design and sperm function. Biology Lett 2: 246-249. doi: 10.1098/ rsbl.2006.0449

17. Mortimer D. 2000. The future of male infertility management and assisted reproduction technology. Hum Reprod 15(Suppl 15): 98-110. doi: 10.1093/ humrep/15.suppl 5.98

18. Muiño R, Fernández M, Areán $H$, Viana JL, López M, Fernández A, Peña AI. 2005. Nuevas tecnologías aplicadas al procesado y evaluación del semen bovino en centros de inseminación artificial. ITEA Información Técnica Económica Agraria 101: 175-191.

19. Muiño R. 2007. Evaluación de la motilidad y viabilidad del semen bovino mediante el uso de sistemas CASA y citometría de flujo: identificación de subpoblaciones espermáticas. Tesis Doc- 
toral. España: Univ. Santiago de Compostela. $157 \mathrm{p}$.

20. Nava-Trujillo H, Quintero-Moreno A. 2017. Distribution of sperm with damaged chromatin among morphometrically distinct subpopulations in cryopreserved semen from brahman bulls. Rev Cient 26: 359-365.

21. Phetudomsinsuk K, Sirinarumitr K, Laikul A, Pinyopummin A. 2008. Morphology and head morphometric characters of sperm in Thai native crossbred stallions. Acta Vet Scand 50: 41. doi: 10.1186/1751-0147-50-41

22. Quintero-Moreno A, Rigau T, Rodríguez-Gil JE. 2004. Regression analyses and motile sperm subpopulation structure study as improving tools in boar semen quality analysis. Theriogenology 61: 673-690. doi: 10.1016/S0093691X(03)00248-6

23. Quintero-Moreno A, Rigau T, Rodríguez-Gil JE. 2007. Multivariate cluster analysis regression procedures as tools to identify motile sperm subpopulations in rabbit semen and to predict semen fertility and litter size. Reprod Domest Anim 42: 312-319. doi: 10.1111/ j.1439-0531.2006.00785.x

24. Rigau T, Farré M, Ballester J, Mogas T, Peña A, Rodríguez-Gil JE. 2001. Effects of glucose and fructose on motility patterns of dog spermatozoa from fresh ejaculates. Theriogenology 56: 801-815. doi: 10.1016/S0093691X(01)00609-4

25. Robayo I, Montenegro V, Valdés C, Cox JF. 2008. CASA assessment of kinematic parameters of ram spermatozoa and their relationship to migration efficiency in ruminant cervical mucus. Reprod Domestic Anim 43: 393-399. doi: 10.1111/j.1439-0531.2007.00920.x

26. Rubio-Guillén J, González D, Garde JJ, Esteso MC, Fernández-Santos MR, Rodríguez-Gil JE, et al. 2007. Effects of cryopreservation on bull spermatozoa distribution in morpho- metrically distinct subpopulations. Reprod Domest Anim 42: 354-357. doi: 10.1111/j.1439-0531.2006.00788.x

27. Sánchez-Partida LG, Windsor DP, Eppleston J, Setchell BP, Maxwell WM. 1999. Fertility and its relationship to motility characteristics of spermatozoa in ewes after cervical, transcervical, and intrauterine insemination with frozenthawed ram semen. J Androl 20: 280288. doi: 10.1002/j.1939-4640.1999.tb02519. $\mathrm{x}$

28. Saravia F, Núñez-Martínez I, Morán $J M$, Soler C, Muriel A, RodríguezMartínez, H, Peña FJ. 2007. Differences in boar sperm head shape and dimensions recorded by computerassisted sperm morphometry are not related to chromatin integrity. Theriogenology 68: 196-203. doi: 10.1016/ j.theriogenology.2007.04.052

29. Tamayo M. 2013. La selección de sementales bovinos en Cuba. 3. Calidad de la producción seminal en futuros sementales Holstein, relación con el desarrollo testicular. REDVET 14(1). [Internet]. Disponible en: http:// www.veterinaria.org/revistas/redvet/ n010113/011301.pdf

30. Thurston LM, Watson PF, Mileham AJ, Holt WV. 2001. Morphologically distinct sperm subpopulations defined by Fourier shape descriptors in fresh ejaculates correlate with variation in boar semen quality following cryopreservation. J Androl 22: 382-294. doi: 10.1002/j.19394640.2001.tb02194.x

31. Verstegen J, Iguer-Ouada M, Onclin K. 2002. Computer assisted semen analyzers in andrology research and veterinary practice. Theriogenology 57 : 149-179. doi: 10.1016/S0093-691X(01)00664-1

32. [World Health Organization] WHO. 1999. Laboratory manual for the examination of human semen and sperm cervical mucus. $4^{\text {th }}$ ed. UK: Cambridge University Press. 128 p. 